\title{
Interacting scalar and spinor fields in Bianchi type I universe filled with magneto-fluid
}

\author{
Bijan Saha \\ Laboratory of Information Technologies \\ Joint Institute for Nuclear Research, Dubna \\ 141980 Dubna, Moscow region, Russid*
}

\begin{abstract}
Self-consistent system of spinor, scalar and BI gravitational fields in presence of magneto-fluid and $\Lambda$-term is considered. Assuming that the expansion of the BI universe is proportional to the $\sigma_{1}^{1}$ component of the shear tensor, exact solutions for the metric functions, as well as for scalar and spinor fields are obtained. For a non-positive $\Lambda$ the initially anisotropic space-time becomes isotropic one in the process of expansion, whereas, for $\Lambda>0$ an oscillatory mode of expansion of the BI model occurs.

PACS numbers: 03.65.Pm and 04.20.Ha

Keywords: Spinor field, Bianchi type I (BI) model, Cosmological constant, Magneto-fluid
\end{abstract}

\section{INTRODUCTION}

The discovery of the cosmic microwave radiation has stimulated a growing interest in anisotropic, general-relativistic cosmological models of the universe. The choice of anisotropic cosmological models in the system of Einstein field equation enable us to study the early day universe, which had an anisotropic phase that approaches an isotropic one [1]. Bianchi type I (BI) cosmological models that are anisotropic homogeneous universes play an important role in understanding essential features of the universe, such as formation of galaxies during its early stage of evolution. An LRS BI model containing a magnetic field directed along one axis with a barotropic fluid was investigated by Thorne [2]. Jacobs [3, 4] investigated BI models with magnetic field satisfying a barotropic equation of state. Bali [5] studied the behavior of the magnetic field in a BI universe for perfect fluid distribution.

In this paper we study the self-consistent system of spinor, scalar and BI gravitational fields in presence of magneto-fluid and cosmological constant. Solutions of Einstein equations coupled to a spinor and a scalar fields in BI spaces have been extensively studied by Saha and Shikin [6, 7, 8, 9]. In the aforementioned papers, we considered spinor field in BI universe where nonlinearity occurred either due self-coupling or induced by an interacting massless scalar field. Here, considering the BI universe filled with magnetofluid we make an attempt to study a system, where all the four fields, i.e., scalar, spinor, electro-magnetic and gravitational ones, play active part in the evolution process.

*Electronic address: saha@thsun1.jinr.ru 


\section{FUNDAMENTAL EQUATIONS AND GENERAL SOLUTIONS}

We choose the action of the self-consistent system of spinor, scalar and gravitational fields in the form

$$
\mathscr{S}(g ; \psi, \bar{\psi}, \varphi)=\int(R+\mathscr{L}) \sqrt{-g} d \Omega
$$

where $R$ is the Ricci scalar and $L$ is the spinor and scalar field Lagrangian density chosen in the form [6]

$$
\mathscr{L}=\frac{i}{2}\left[\bar{\psi} \gamma^{\mu} \nabla_{\mu} \psi-\nabla_{\mu} \bar{\psi} \gamma^{\mu} \psi\right]-m \bar{\psi} \psi+\frac{1}{2} \varphi_{, \alpha} \varphi^{, \alpha}(1+\lambda F)
$$

Here $\lambda$ is the coupling constant and $F$ is some arbitrary functions of invariants generated from the real bilinear forms of a spinor field. We choose $F$ to be the function of $I=S^{2}=$ $(\bar{\psi} \psi)^{2}$ and $J=P^{2}=\left(i \bar{\psi} \gamma^{5} \psi\right)^{2}$, i.e., $F=F(I, J)$, that describes the nonlinearity in the most general of its form [8]. As one sees, for $\lambda=0$ we have the system with minimal coupling.

The gravitational field in our case is given by a Bianchi type I (BI) metric in the form

$$
d s^{2}=a_{0}^{2}\left(d x^{0}\right)^{2}-a_{1}^{2}\left(d x^{1}\right)^{2}-a_{2}^{2}\left(d x^{2}\right)^{2}-a_{3}^{2}\left(d x^{3}\right)^{2},
$$

with $a_{0}=1, x^{0}=c t$ and $c=1$. The metric functions $a_{i}(i=1,2,3)$ are the functions of time $t$ only.

Variation of (2.1) with respect to spinor field $\psi(\bar{\psi})$ gives nonlinear spinor field equations

$$
\begin{aligned}
& i \gamma^{\mu} \nabla_{\mu} \psi-m \psi+\mathscr{D} \psi+\mathscr{G}_{i} \gamma^{5} \psi=0 \\
& i \nabla_{\mu} \bar{\psi} \gamma^{\mu}+m \bar{\psi}-\mathscr{D} \bar{\psi}-\mathscr{G} i \bar{\psi} \gamma^{5}=0
\end{aligned}
$$

where we denote

$$
\mathscr{D}=\lambda S \varphi_{, \alpha} \varphi^{, \alpha} \partial F / \partial I, \quad \mathscr{G}=\lambda P \varphi_{, \alpha} \varphi^{, \alpha} \partial F / \partial J,
$$

whereas, variation of (2.1) with respect to scalar field yields the following scalar field equation

$$
\frac{1}{\sqrt{-g}} \frac{\partial}{\partial x^{v}}\left(\sqrt{-g} g^{v \mu}(1+\lambda F) \varphi, \mu\right)=0 .
$$

Varying (2.1) with respect to metric tensor $g_{\mu \nu}$ one finds the gravitational field equation which in account of cosmological constant $\Lambda$ has the form

$$
\begin{aligned}
\frac{\ddot{a}_{2}}{a_{2}}+\frac{\ddot{a}_{3}}{a_{3}}+\frac{\dot{a}_{2}}{a_{2}} \frac{\dot{a}_{3}}{a_{3}} & =\kappa T_{1}^{1}-\Lambda, \\
\frac{\ddot{a}_{3}}{a_{3}}+\frac{\ddot{a}_{1}}{a_{1}}+\frac{\dot{a}_{3}}{a_{3}} \frac{\dot{a}_{1}}{a_{1}} & =\kappa T_{2}^{2}-\Lambda, \\
\frac{\ddot{a}_{1}}{a_{1}}+\frac{\ddot{a}_{2}}{a_{2}}+\frac{\dot{a}_{1}}{a_{1}} \frac{\dot{a}_{2}}{a_{2}} & =\kappa T_{3}^{3}-\Lambda, \\
\frac{\dot{a}_{1}}{a_{1}} \frac{\dot{a}_{2}}{a_{2}}+\frac{\dot{a}_{2}}{a_{2}} \frac{\dot{a}_{3}}{a_{3}}+\frac{\dot{a}_{3}}{a_{3}} \frac{\dot{a}_{1}}{a_{1}} & =\kappa T_{0}^{0}-\Lambda .
\end{aligned}
$$


Here $\kappa$ is the Einstein gravitational constant and over-dot means differentiation with respect to $t$. The energy-momentum tensor of the system is given by

$$
\begin{aligned}
T_{\mu}^{\rho}= & \frac{i}{4} g^{\rho v}\left(\bar{\psi} \gamma_{\mu} \nabla_{\nu} \psi+\bar{\psi} \gamma_{\nu} \nabla_{\mu} \psi-\nabla_{\mu} \bar{\psi} \gamma_{\nu} \psi-\nabla_{\nu} \bar{\psi} \gamma_{\mu} \psi\right) \\
& +(1-\lambda F) \varphi_{, \mu} \varphi^{\rho}-\delta_{\mu}^{\rho} \mathscr{L}+T_{\mathrm{m} \mu}^{v} .
\end{aligned}
$$

The energy-momentum tensor of the magneto-fluid is chosen to be

$$
T_{\mu(m)}^{v}=(\varepsilon+p) v_{\mu} v^{v}-p \delta_{\mu}^{v}+E_{\mu}^{v},
$$

where $E_{\mu \nu}$ is the electro-magnetic field given by Lichnerowich [10]

$$
E_{\mu}^{v}=\bar{\mu}\left[|h|^{2}\left(u_{\mu} u^{v}-\frac{1}{2} \delta_{\mu}^{v}\right)-h_{\mu} h^{v}\right] .
$$

Here $u^{\mu}$ is the flow vector satisfying

$$
g_{\mu v} u^{\mu} u^{v}=1,
$$

$\bar{\mu}$ is the magnetic permeability and $h_{\mu}$ is the magnetic flux vector defined by

$$
h_{\mu}=\frac{1}{\bar{\mu}} \star F_{v \mu} u^{v}
$$

where $\star F_{\mu \nu}$ is the dual electro-magnetic field tensor defined as

$$
\star F_{\mu v}=\frac{\sqrt{-g}}{2} \varepsilon_{\mu v \alpha \beta} F^{\alpha \beta}
$$

Here $F^{\alpha \beta}$ is the electro-magnetic field tensor and $\varepsilon_{\mu v \alpha \beta}$ is the totally anti-symmetric LeviCivita tensor with $\varepsilon_{0123}=+1$. Here the comoving coordinates are taken to be $u^{0}=1, u^{1}=$ $u^{2}=u^{3}=0$. We choose the incident magnetic field to be in the direction of $x$-axis so that the magnetic flux vector has only one nontrivial component, namely $h_{1} \neq 0$. In view of the aforementioned assumption from (2.11) we obtain $F_{12}=F_{13}=0$. We also assume that the conductivity of the fluid is infinite. This leads to $F_{01}=F_{02}=F_{03}=0$. Thus we have only one non-vanishing component of $F_{\mu \nu}$ which is $F_{23}$. Then from the first set of Maxwell equation

$$
F_{\mu v ; \beta}+F_{v \beta ; \mu}+F_{\beta \mu ; \nu}=0
$$

where the semicolon stands for covariant derivative, one finds

$$
F_{23}=\mathscr{I}, \quad \mathscr{I}=\text { const. }
$$

Then from (2.11) in account of (2.12) one finds

$$
h_{1}=\frac{a_{1} \mathscr{I}}{\bar{\mu} a_{2} a_{3}} .
$$

Finally, for $E_{\mu}^{v}$ we find the following non-trivial components

$$
E_{0}^{0}=E_{1}^{1}=-E_{2}^{2}=-E_{3}^{3}=\frac{\mathscr{I}^{2}}{2 \bar{\mu} a_{2}^{2} a_{3}^{2}} .
$$


In (2.8) $\varepsilon$ and $p$ are the energy and pressure of the fluid, respectively. In this note we assume that the equation of state

$$
p=\zeta \varepsilon
$$

holds. Here $\zeta$ varies between the interval $0 \leq \zeta \leq 1$, whereas $\zeta=0$ describes the dust Universe, $\zeta=\frac{1}{3}$ presents radiation Universe, $\frac{1}{3}<\zeta<1$ ascribes hard Universe and $\zeta=1$ corresponds to the stiff matter. The Dirac matrices $\gamma_{\mu}(x)$ of curve space-time are connected with those of Mincowski space as

$$
\gamma^{\mu}=\bar{\gamma}^{\mu} / a_{\mu}, \quad \gamma_{\mu}=\bar{\gamma} a_{\mu}, \quad \mu=0,1,2,3 .
$$
11]

In the Eqs. (2.4) and (2.7) $\nabla_{\mu}$ is the covariant derivatives acting on a spinor field as

$$
\nabla_{\mu} \psi=\frac{\partial \psi}{\partial x^{\mu}}-\Gamma_{\mu} \psi, \quad \nabla_{\mu} \bar{\psi}=\frac{\partial \bar{\psi}}{\partial x^{\mu}}+\bar{\psi} \Gamma_{\mu}
$$

where $\Gamma_{\mu}$ are the Fock-Ivanenko spinor connection coefficients defined by

$$
\Gamma_{\mu}=\frac{1}{4} \gamma^{\sigma}\left(\Gamma_{\mu \sigma}^{v} \gamma_{v}-\partial_{\mu} \gamma_{\sigma}\right)
$$

For the metric (2.3) one has the following components of the spinor connection coefficients

$$
\Gamma_{\mu}=(1 / 2) \dot{a}_{\mu} \bar{\gamma}^{\mu} \bar{\gamma}^{0}
$$

We study the space-independent solutions to the spinor and scalar field Eqs. (2.4) and (2.5) so that $\psi=\psi(t)$ and $\varphi=\varphi(t)$. defining

$$
\tau=a_{0} a_{1} a_{2} a_{3}=\sqrt{-g}
$$

from (2.5) for the scalar field we have

$$
\varphi=C \int[\tau(1+\lambda F)]^{-1} d t
$$

Setting $V_{j}(t)=\sqrt{\tau} \psi_{j}(t), \quad j=1,2,3,4$, in view of (2.19) and (2.21) from (2.4a) one deduces the following system of equations:

$$
\begin{aligned}
& \dot{V}_{1}+i(m-\mathscr{D}) V_{1}-\mathscr{G} V_{3}=0, \\
& \dot{V}_{2}+i(m-\mathscr{D}) V_{2}-\mathscr{G} V_{4}=0, \\
& \dot{V}_{3}-i(m-\mathscr{D}) V_{3}+\mathscr{G} V_{1}=0, \\
& \dot{V}_{4}-i(m-\mathscr{D}) V_{4}+\mathscr{G} V_{2}=0 .
\end{aligned}
$$

From (2.4a) we also write the equations for the bilinear spinor forms $S, \quad P$ and $A^{0}=$ $\bar{\psi} \bar{\gamma}^{5} \bar{\gamma}^{0} \psi$

$$
\begin{aligned}
\dot{S}_{0}-2 \mathscr{G} A_{0}^{0} & =0, \\
\dot{P}_{0}-2(m-\mathscr{D}) A_{0}^{0} & =0, \\
\dot{A}_{0}^{0}+2(m-\mathscr{D}) P_{0}+2 \mathscr{G} S_{0} & =0,
\end{aligned}
$$

where $Q_{0}=\tau Q$, leading to the relation $S^{2}+P^{2}+\left(A^{0}\right)^{2}=C^{2} / \tau^{2}, \quad C^{2}=$ const. As one sees, for $F=F(I)$ (2.25a) gives $S=C_{0} / \tau$, whereas for the massless spinor field with $F=F(J)$ 
(2.25b) yields $P=D_{0} / \tau$. In view of it for $F=F(I)$ we obtain the following expression for the components of spinor field

$$
\begin{aligned}
& \psi_{1}(t)=C_{1} \tau^{-1 / 2} e^{-i \beta}, \quad \psi_{2}(t)=C_{2} \tau^{-1 / 2} e^{-i \beta}, \\
& \psi_{3}(t)=C_{3} \tau^{-1 / 2} e^{i \beta}, \quad \psi_{4}(t)=C_{4} \tau^{-1 / 2} e^{i \beta},
\end{aligned}
$$

with $C_{i}$ being the integration constants and are related to $C_{0}$ as $C_{0}=C_{1}^{2}+C_{2}^{2}-C_{3}^{2}-C_{4}^{2}$. Here $\beta=\int(m-\mathscr{D}) d t$. In case of $F=F(J)$ for the massless spinor field we get

$$
\begin{array}{ll}
\psi_{1}=\tau^{-1 / 2}\left(D_{1} e^{i \sigma}+i D_{3} e^{-i \sigma}\right), & \psi_{2}=\tau^{-1 / 2}\left(D_{2} e^{i \sigma}+i D_{4} e^{-i \sigma}\right), \\
\psi_{3}=\tau^{-1 / 2}\left(i D_{1} e^{i \sigma}+D_{3} e^{-i \sigma}\right), & \psi_{4}=\tau^{-1 / 2}\left(i D_{2} e^{i \sigma}+D_{4} e^{-i \sigma}\right) .
\end{array}
$$

The integration constants $D_{i}$ are connected to $D_{0}$ by $D_{0}=2\left(D_{1}^{2}+D_{2}^{2}-D_{3}^{2}-D_{4}^{2}\right)$. Here we set $\sigma=\int \mathscr{G} d t$.

Once the spinor functions are known explicitly, one can write the components of spinor current $j^{\mu}=\bar{\psi} \gamma^{\mu} \psi$, the charge density of spinor field $\rho=\left(j_{0} \cdot j^{0}\right)^{1 / 2}$, the total charge of spinor field $Q=\int_{-\infty}^{\infty} \rho \sqrt{-3} d x d y d z$, the components of spin tensor $S^{\mu v, \varepsilon}=\frac{1}{4} \bar{\psi}\left\{\gamma^{\varepsilon} \sigma^{\mu v}+\right.$ $\left.\sigma^{\mu v} \gamma^{\varepsilon}\right\} \psi$ and other physical quantities.

Let us now solve the Einstein equations. In doing so we first write the expressions for the components of the energy-momentum tensor explicitly:

$$
\begin{aligned}
& T_{0}^{0}=m S+C^{2} / 2 \tau^{2}(1+\lambda F)+\varepsilon+\frac{\mathscr{I}^{2}}{2 \bar{\mu} a_{2}^{2} a_{3}^{2}}, \\
& T_{1}^{1}=\mathscr{D} S+\mathscr{G} P-C^{2} / 2 \tau^{2}(1+\lambda F)-p+\frac{\mathscr{I}^{2}}{2 \bar{\mu} a_{2}^{2} a_{3}^{2}}, \\
& T_{2}^{2}=\mathscr{D} S+\mathscr{G} P-C^{2} / 2 \tau^{2}(1+\lambda F)-p-\frac{\mathscr{I}^{2}}{2 \bar{\mu} a_{2}^{2} a_{3}^{2}}, \\
& T_{3}^{3}=\mathscr{D} S+\mathscr{G} P-C^{2} / 2 \tau^{2}(1+\lambda F)-p-\frac{\mathscr{I}^{2}}{2 \bar{\mu} a_{2}^{2} a_{3}^{2}},
\end{aligned}
$$

In view of $T_{2}^{2}=T_{3}^{3}$ from (2.6b), (2.6c) we find

$$
a_{2}=a_{3} D \exp \left(X \int \frac{d t}{\tau}\right)
$$

with the constants of integration $D$ and $X$ being integration constants.

Following Bali [5] let us assume that the expansion $(\boldsymbol{\theta})$ in the model is proportional to the eigenvalue $\sigma_{1}^{1}$ of the shear tensor $\sigma_{\mu}^{v}$. Since for the BI space-time

$$
\begin{aligned}
\theta & =\frac{\dot{a}_{1}}{a_{1}}+\frac{\dot{a}_{2}}{a_{2}}+\frac{\dot{a}_{3}}{a_{3}}, \\
\sigma_{1}^{1} & =-\frac{1}{3}\left(4 \frac{\dot{a}_{1}}{a_{1}}+\frac{\dot{a}_{2}}{a_{2}}+\frac{\dot{a}_{3}}{a_{3}}\right),
\end{aligned}
$$


the aforementioned condition leads to

$$
a_{1}=\left(a_{2} a_{3}\right)^{N}
$$

with $N$ being the proportionality constant.

In account of (2.22) from (2.29) and (2.32) after some manipulation for the metric functions one finds

$$
\begin{aligned}
& a_{1}=\tau^{N /(N+1)} \\
& a_{2}=\sqrt{D} \tau^{1 / 2(N+1)} \exp \left[\frac{X}{2} \int \frac{d t}{\tau}\right], \\
& a_{3}=\frac{1}{\sqrt{D}} \tau^{1 / 2(N+1)} \exp \left[-\frac{X}{2} \int \frac{d t}{\tau}\right] .
\end{aligned}
$$

As one sees from (2.33) for $\tau \sim t^{n}$ with $n>1$ the exponent tends to unity at large $t$. In this case the anisotropic model becomes isotropic one iff $D=1$ and $N=1 / 2$. Let us also write the invariants of gravitational field. They are the Ricci scalar $I_{1}=R \approx 1 / \tau^{2}$, $I_{2}=R_{\mu \nu} R^{\mu v} \equiv R_{\mu}^{v} R_{v}^{\mu} \approx 1 / \tau^{4}$ and the Kretschmann scalar $I_{3}=R_{\alpha \beta \mu \nu} R^{\alpha \beta \mu \nu} \approx 1 / \tau^{4}$. As we see, the space-time becomes singular at a point where $\tau=0$, as well as the scalar and spinor fields. Thus we see, all the functions in question are expressed via $\tau$. In what follows, we write the equation for $\tau$ and study it in details.

Summation of Einstein Eqs. (2.6a), (2.6b), (2.6c) and (2.6d) multiplied by 3 gives

$$
\frac{\ddot{\tau}}{\tau}=\frac{3}{2} \kappa\left(m S+\mathscr{D} S+\mathscr{G} P+\varepsilon-p+\frac{2 \mathscr{I}^{2}}{3 \bar{\mu}\left(a_{2} a_{3}\right)^{2}}\right)-3 \Lambda .
$$

For the right hand side of Eq. (2.34) to be a function of $\tau$ only, the solution to this equation is well known [12]. In what follows we study this equation for some concrete form of $F$. In doing so let us demand the energy-momentum to be conserved, i.e., $T_{\mu ; v}^{v}=0$, which in our case takes the form

$$
\frac{1}{\tau}\left(\tau T_{0}^{0}\right)^{\cdot}-\frac{\dot{a}_{1}}{a_{1}} T_{1}^{1}-\frac{\dot{a}_{2}}{a_{2}} T_{2}^{2}-\frac{\dot{a}_{3}}{a_{3}} T_{3}^{3}=0
$$

In account of the equation of state (2.17) and

$$
(m-\mathscr{D}) \dot{S}_{0}-\mathscr{G} \dot{P}_{0}=0
$$

which follows from (2.25), after a little manipulation from (2.35) we obtain

$$
\varepsilon=\varepsilon_{0} / \tau^{1+\zeta}, \quad p=\zeta \varepsilon_{0} / \tau^{1+\zeta}
$$

Let us recall that we consider $F$ as a function of $I, J$ or $I \pm J$. If we choose $F=F(I)$, then setting $m=0$ we come to the analogical equation corresponding to the massless spinor case with $F=F(J)$ or $F=F(I \pm J)$, whereas setting $\lambda=0$ we have the system with minimal coupling. Under this assumption from (2.25) one finds

$$
S=\frac{C_{0}}{\tau}
$$


In view of (2.37), (2.36) and the fact that $a_{2} a_{3}=\tau^{1 /(N+1)}$, Eq. (2.34) can be rewritten as

$$
\ddot{\tau}=\frac{3}{2} \kappa\left(m C_{0}+\mathscr{D} C_{0}+\varepsilon_{0}(1-\zeta) / \tau^{\zeta}+\frac{2 \mathscr{I}^{2}}{3 \bar{\mu}} \tau^{(N-1) /(N+1)}\right)-3 \Lambda .
$$

Recalling the definition of $\mathscr{D}$ the solution to Eq. (2.38) can be written in quadrature

$$
\int \frac{d \tau}{\sqrt{\kappa\left(m C_{0} \tau+C^{2} / 2(1+\lambda F)+\varepsilon_{0} \tau^{1-\zeta}+\left((N+1) \mathscr{I}^{2} / 3 \bar{\mu} N\right) \tau^{2 N /(N+1)}\right)-\Lambda \tau^{2}+E}}=\sqrt{3} t
$$

with $E$ being some integration constant. It should be mentioned that being the volumescale $\tau$ is non-negative. At the points where $\tau=0$ there occurs space-time singularity. On the other hand, the radical in (2.39) should be positive. This fact leads to the conclusion that for $\Lambda>0$ the value of $\tau$ is bound from above as well, giving rise to an oscillatory mode of expansion of the BI universe. For a non-positive $\Lambda$, we have picture with fast expanding $\tau$ with time.

\section{CONCLUSIONS}

A self-consistent system of spinor, scalar and gravitation fields has been studied in presence of magneto-fluid and cosmological term $\Lambda$. With the presence of $F_{23}$ component of electro-magnetic field tensor, the system can be viewed as one where all the four fields, i.e., scalar, electro-magnetic, spinor and gravitational ones, are taken into consideration. Assuming that the expansion of the $\mathrm{BI}$ space-time is proportional to the $\sigma_{1}^{1}$ component of the shear tensor, solutions for the metric functions $a_{i}(t)$ are obtained explicitly in terms of volume-scale $\tau$. Expressions for the scalar and spinor fields are also obtained in terms of $\tau$. For the non-positive $\Lambda$ we obtain exponentially expanding BI universe, which means the initially anisotropic space-time becomes isotropic one in the process of expansion. For a positive $\Lambda$ an oscillatory mode of expansion takes place. Choosing the integration constant $E$ and initial value value of $\tau$ it is possible to obtain solutions those are regular everywhere. A detailed numerical study of the Eq. (2.38) we plan to perform in short.

Acknowledgments

[1] Misner C.W. Astrophysical Journal 151, 431 (1968).

[2] Thorne K.S. Astrophysical Journal 148, 51 (1967).

[3] Jacobs K.C. Astrophysical Journal 153, 661 (1968).

[4] Jacobs K.C. Astrophysical Journal 155, 379 (1969).

[5] Bali R. International Journal of Theoretical Physics 25, 755 (1986).

[6] Saha B., Shikin G.N. General Relativity and Gravitation 29, 1099 (1997).

[7] Saha B., Shikin G.N. Journal Mathematical Physics 38, 5305 (1997).

[8] Bijan Saha Physical Review D 64, 123501 (2001).

[9] Bijan Saha Modern Physics Letters A 16, 1287 (2001). 
[10] Lichnerowicz A. Relativistic Hydrodynamics and Magnetohydrodynamics, (Benjamin, New York)

[11] Brill D. and Wheeler J. Review of Modern Physics 29, 465 (1957).

[12] E. Kamke, Differentialgleichungen losungsmethoden und losungen (Akademische Verlagsgesellschaft, Leipzig, 1957). 